\title{
SDO/AIA and Hinode/EIS observations of interaction between an EUV wave and active region loops
}

\author{
Liheng Yang ${ }^{1}$, Jun Zhang ${ }^{1}$, Ting $\mathbf{L i}^{1}$ and Wei Liu ${ }^{2}$ \\ ${ }^{1}$ Key Laboratory of Solar Activity, National Astronomical Observatories, \\ Chinese Academy of Sciences, Beijing 100012, China \\ email: yangliheng@bao.ac.cn \\ ${ }^{2}$ W. W. Hansen Experimental Physics Laboratory, Stanford University, Stanford, \\ CA 94305, USA
}

\begin{abstract}
We study an EUV wave initiated in active region (AR) 11261 on 2011 August 4 by using the SDO/AIA and the Hinode/EIS. We found that: (1) the EUV wave interacted with AR loops between AR 11261 and AR 11263, and exited mass flows in these loops. (2) EIS observations of AR loops in AR 11263 revealed that at the time of the wave transit, the original red-shifted feature had an increase of about $3 \mathrm{~km} \mathrm{~s}^{-1}$, and the original blue-shifted feature slightly weakened. These findings could be explained in the framework of a fast-mode magnetosonic wave interpretation for EUV waves.
\end{abstract}

Keywords. Sun: activity, Sun: corona, Sun: flares

\section{Observations}

On 2011 August 4, the AR 11261 produced an M9.3 class flare at the location of $\mathrm{N} 16^{\circ} \mathrm{W} 49^{\circ}$, which started at 03:41 UT and peaked at 03:57 UT. Following the onset of the flare, we observed an EUV wave. For studying the dynamics of the EUV wave, we get the time-distance slices along "A" as shown in Figure 1(a). From this slice (see Figure 1(b)), we note that this wave initially moved through the AR loops connecting AR 11261 and AR 11263 (marked by "L1" in Figure 1(a)) with a velocity of $486 \pm 9 \mathrm{~km} \mathrm{~s}^{-1}$. At 04:00:24 UT, the wave arrived at the apex of the L1, and excited mass flows in the L1.
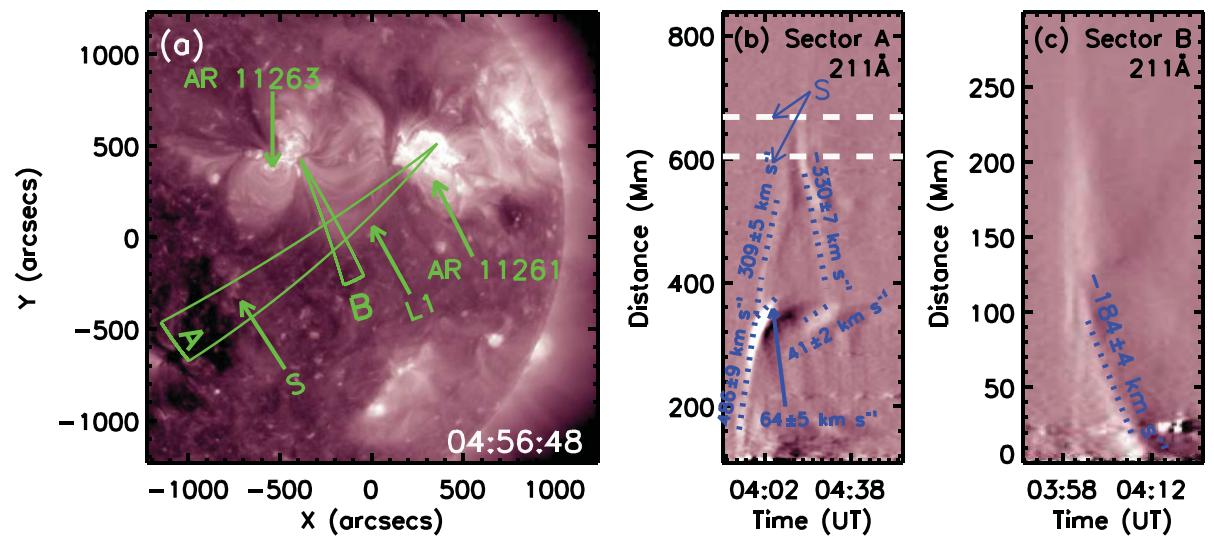

Figure 1. (a) An SDO/AIA $211 \AA$ direct image showing two $10^{\circ}$ wide sectors ("A" and "B"), which are used to obtained running difference space-time plots in (b) and (c). 
From the time-distance plot of Sector "B" (see Figure 1(a) and (c)), we note that the mass flows gradually injected into the footpoints of $\mathrm{L} 1$, with a velocity of about $184 \pm 4$ $\mathrm{kms}^{-1}$. Simultaneously, the wave pushed the L1 forward with a velocity of about $64 \pm 5$ $\mathrm{km} \mathrm{s}^{-1}$. This movement lasted about $10 \mathrm{~min}$, inducing a maximum displacement of about $36 \mathrm{Mm}$. About $14 \mathrm{~min}$ later, another moving bright feature appeared at the apex of the L1 (see Figure 1(b)). It had a lower velocity of about $41 \pm 2 \mathrm{~km} \mathrm{~s}^{-1}$, but lasted a much longer time (about $15 \mathrm{~min}$ ). When the coronal wave encountered a small-scale coronal structure (marked by "S" in Figure 1(a) and denoted by white lines in Figure 1(b)), a reflected wave appeared (see Figure 1(b)). The reflected wave had a velocity of $330 \pm 7$ $\mathrm{km} \mathrm{s}^{-1}$, which was a little higher than that of the incident wave (about $309 \pm 5 \mathrm{~km} \mathrm{~s}^{-1}$ ).

At about 04:01 UT, the east part of the EUV wave propagated to AR $11263\left(\mathrm{~N} 17^{\circ} \mathrm{W} 18^{\circ}\right)$ and was captured by Hinode/EIS. Figure 2 shows the Doppler velocity and line width for the Fe XII $195.12 \AA$ line as a function of time. We note that when the coronal wave transit, some red-shifted features appeared or enhanced, and the corresponding line width obviously broadened. To quantitatively analyze these features, we calculated the averaged Doppler velocity and line width in areas "A" and "B". At the time of the EUV wave transit (marked by the dashed lines in Figure 2(b) and (d)), the red-shifted feature in "A" increased by about $3 \mathrm{~km} \mathrm{~s}^{-1}$ and formed a sharp peak (marked by "vp" in Figure $2(\mathrm{~b})$ ), while the blue-shifted feature in "B" decreased to $\sim 2 \mathrm{kms}^{-1}$ and formed an unconspicuous peak. There also existed a sharp peak (marked by "wp" in Figure 2(d)) in the time profile of the line width. We note that the line width increased by about 7 $\mathrm{m} \AA$ in " $\mathrm{A}$ " and $5 \mathrm{~m} \AA$ in "B".

\section{Conclusions}

Our observational results are consistent with the fast-mode magnetosonic wave interpretation for EUV waves. The wave disturbed the plasma at the apex of the AR loops connecting AR 11261 and AR 11263, and generated mass flows along these loops. In addition, the wave compressed the plasma of the AR loops in AR 11263, and induced that the original red-shifted feature increased, and the original blue-shifted feature slightly weakened.
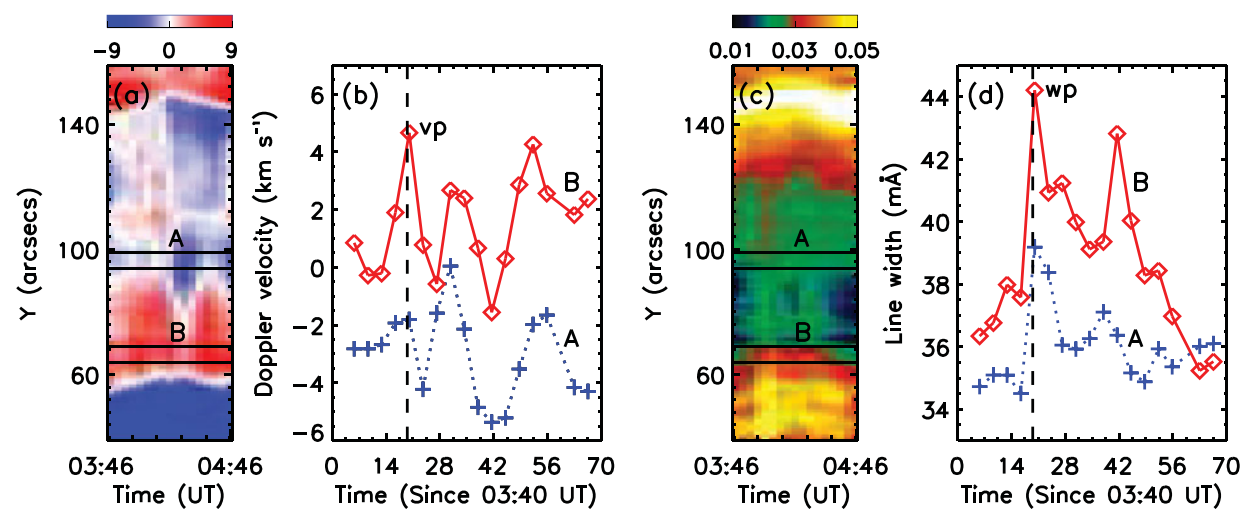

Figure 2. Doppler velocity (a) and line width (c) for the Fe XII 195.12 line as a function of time. Time profiles of averaged Doppler velocity (b) and line width (d) (calculated within "A" and "B" in this figure). 